\title{
Pristimerin protects against doxorubicin-induced cardiotoxicity and fibrosis through modulation of Nrf2 and MAPK/NF-kB signaling pathways
}

This article was published in the following Dove Press journal: Cancer Management and Research

\author{
Dina S El-Agamy ${ }^{1,2}$ \\ Khaled M El-Harbi ${ }^{3}$ \\ Saad Khoshhal ${ }^{3}$ \\ Nishat Ahmed' \\ Mohamed A Elkablawy 4,5 \\ Ahmed A Shaaban ${ }^{2,6}$ \\ Hany M Abo-Haded ${ }^{3,7}$ \\ 'Department of Pharmacology and \\ Toxicology, College of Pharmacy, \\ Taibah University, Al-Madinah \\ Al-Munawwarah 3000I, Saudi Arabia; \\ ${ }^{2}$ Department of Pharmacology and \\ Toxicology, Faculty of Pharmacy, \\ Mansoura University, Mansoura \\ 355 I6, Egypt; ${ }^{3}$ Cardiogenetic Team, \\ Department of Pediatrics, College \\ of Medicine, Taibah University, \\ Al-Madinah Al-Munawwarah 3000I, \\ Saudi Arabia; ${ }^{4}$ Department of \\ Pathology, Faculty of Medicine, \\ Taibah University, Al-Madinah \\ Al-Munawwarah 3000I, Saudi Arabia; \\ ${ }^{5}$ Department of Pathology, Faculty \\ of Medicine, Menoufia University, \\ Menoufia 325I I, Egypt; ' ${ }^{\circ}$ epartment \\ of Pharmacology, Faculty of Pharmacy, \\ Aqaba University of Technology, Aqaba \\ 77II 0, Jordan; ${ }^{7}$ Pediatric Cardiology \\ Unit, Department of Pediatrics, \\ Faculty of Medicine, Mansoura \\ University, Mansoura 355 I6, Egypt
}

Correspondence: Hany M Abo-Haded Pediatric Cardiology Unit, Department of Pediatrics, Faculty of Medicine, Mansoura University, Elgomhouriya Street,

Mansoura, Dakahlia Governorate, 355I6, Egypt

Tel +20 I00 I90 I727

Email hany_haded@yahoo.com
Background/purpose: Pristimerin (Pris) is triterpenoid compound with many biological effects. Until now, nothing is known about its effect on doxorubicin (DOX)-induced cardiotoxicity. Hence, this study investigated the impact of Pris on DOX-induced cardiotoxic effects. Materials and methods: Rats were treated with Pris 1 week before and 2 weeks contaminant with repeated DOX injection. Afterwards, electrocardiography (ECG), biochemical, histopathological, PCR, and Western blot assessments were performed.

Results: Pris effectively alleviated DOX-induced deleterious cardiac damage. It inhibited DOXinduced ECG abnormities as well as DOX-induced elevation of serum indices of cardiotoxicity. The histopathological cardiac lesions and fibrosis were remarkably improved in Pris-treated animals. Pris reduced hydroxyproline content and attenuated the mRNA and protein expression of the pro-fibrogenic genes. The antioxidant activity of Pris was prominent through the amelioration of oxidative stress parameters and enhancement of antioxidants. Furthermore, Pris enhanced the activation of nuclear factor-erythroid 2 related factor 2 (Nrf2) signaling pathway as it increased the mRNA and protein expression of Nrf2 and Nrf2-dependent antioxidant genes (GCL, NQO1, HO-1). Additionally, the anti-inflammatory effect of Pris was obvious through the inhibition of mitogen activated protein kinase (MAPK)/nuclear factor kappa-B (NF- $\mathrm{KB})$ signaling and subsequent inhibition of inflammatory mediators.

Conclusion: This study provides evidence of the cardioprotective activity of Pris which is related to the modulation of Nrf2 and MAPK/NF- $\mathrm{KB}$ signaling pathways.

Keywords: doxorubicin, pristimerin, cardiotoxicity, Nrf2, MAPK/NF- $\mathrm{BB}$

\section{Introduction}

Doxorubicin (DOX) is a broad spectrum anthracycline antineoplastic agent that is used in various malignancies. Its antitumor action is mediated through inhibition of topoisomerase-II enzyme, DNA damage, prevention of DNA repair, and cell replication which lead to cell death in highly proliferative tissues. ${ }^{1}$ Treatment with DOX has been associated with fatal cardiotoxic adverse effects which may range from pericarditis, transient controllable arrhythmias, and reversible hypotension observed immediately after treatment to congestive heart failure with mortality rate exceeding $50 \%$ in case of chronic treatment. ${ }^{2}$ Chronic use of DOX produces cardiac fibrosis and remodeling. This is probably due to the imbalance between the synthesis and degradation of extracellular matrix (ECM) collagens. Alteration in ECM usually results in left ventricle dysfunction and progression of heart failure..$^{3-5}$

The exact molecular mechanisms underlying DOX-induced cardiotoxicity are complex and still not fully clarified. Studies have proposed that oxidative stress, 
inflammation, and apoptosis are the main players that control DOX pathogenesis. ${ }^{6}$ Excessive generation of free radicals during DOX metabolism augments oxidative stress, lipid peroxidation, membrane damage, and cardiomyocyte death. ${ }^{1}$ The nuclear factor-erythroid 2 related factor 2 (Nrf2) is a transcription factor that controls the expression of enzymes involved in the anti-oxidative stress response and hence modulates the cellular defense pathway. ${ }^{7}$ Recent studies have shown that Nrf2 activation could counteract DOX-induced cardiotoxicity via suppression of DOX-induced oxidative stress and impairment of autophagy. ${ }^{8,9}$

Activation of cellular inflammatory pathways is greatly involved in DOX-induced cardiac injury. DOX activates

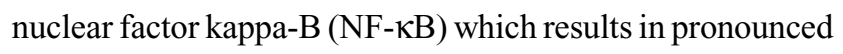
inflammatory reactions and cellular damage. ${ }^{10}$ Inhibition of $\mathrm{NF}-\kappa \mathrm{B}$ pathway by different agents was shown to confer protection against DOX-induced cardiotoxicity. ${ }^{11-13}$ Even though DOX is still used, only few chemicals are adopted to protect the heart against deleterious adverse effects of DOX. Hence, efforts must continue to find new therapeutics that can oppose DOX-induced cardiotoxicity.

Various active compounds derived from medicinal plants have been considered as an attractive alternative approach for multiple organ dysfunction. Pristimerin (Pris) is a natural triterpenoid compound (Figure 1A). It is extracted from Celastraceae plants and has been used in Chinese traditional medicine because of its multiple biological properties. Studies have demonstrated that Pris can be an effective therapeutic for different tumors, and autoimmune and inflammatory disorders. ${ }^{14,15}$ The anti-inflammatory activity of Pris was initially linked to suppression of the release of nitric oxide (NOx) via inhibition of inducible nitric oxide synthase (iNOS). More recently, Pris was shown to inhibit NF- $\kappa \mathrm{B}$ and its inflammatory cascade. ${ }^{16,17}$ Based on these data, we hypothesized that Pris might have potential protective activities against DOXinduced myocardial damage. The current study evaluated the effects of Pris treatment on DOX-induced cardiac injury and fibrosis in rats. Furthermore, the impact of Pris on different molecular pathways was assessed.

\section{Materials and methods Drugs and reagents}

DOX was purchased as ampoules (Ebewe Pharma GmbH, Austria). Pris was purchased from Tocris Bioscience (Bristol, UK) and dissolved in dimethyl sulfoxide (DMSO). Ketamine was obtained as ampoules (Tekam; Hikma Pharmaceuticals, Amman, Jordan). Creatine kinase isoenzyme-MB (CK-MB) kit was obtained from ELITech, France. Lactate dehydrogenase (LDH) activity kit was purchased from Human Biochemica und Diagnostica GmbH (Wiesbaden, Germany). ELISA kits for troponin I (cTnI) and troponin $\mathrm{T}$ (cTnT) were purchased from Kamiya Biomedical Co (Tukwila, WA, USA). Hydroxyproline colorimetric assay kit was purchased from Abcam (Cambridge, UK). Malondialdehyde (MDA), superoxide dismutase (SOD), reduced glutathione (GSH) kits were purchased from Biodiagnostic Company (Giza, Egypt). ELISA kits for 4-hydroxynonenal (4-HNE), 8-hydroxy-2-deoxyguanosine (8-OHdG), and protein carbonyl (PC) were purchased from MyBioSource Inc. (San Diego, CA, USA). Nuclear extraction and NF- $\kappa \mathrm{B}$ p65 activation kits were bought from Abcam. ELISA kits for NOx, tumor necrosis factor- $\alpha$ (TNF- $\alpha$ ), and interleukin-6 (IL-6) were purchased from R\&D Systems, Minneapolis, $\mathrm{MN}$, USA. Total protein extraction kit was purchased from KeyGEN Biotech Co, Ltd (Nanjing, China). Bicinchoninic acid (BCA) Protein Assay Kit was purchased from Thermo Fisher Scientific Inc. (Waltham, MA, USA).

Primary antibodies were purchased from Abcam and Thermo Fisher Scientific Inc. for the following proteins: transforming growth factor-beta1 (TGF- $\beta 1$, cat no ab92486), metalloproteinase-2 (MMP-2, cat no ab37150), metalloproteinase-9 (MMP-9, cat no ab38898), tissue inhibitor of metalloproteinase-1 (TIMP-1, cat no ab61224), Nrf2 (cat no ab137550), glutamate-cysteine ligase catalytic subunit (GCLc, cat no ab80841), NAD(P)H dehydrogenase (quinone) 1 (NQO1, cat no ab28947), heme-oxygenase-1 (HO-1, cat

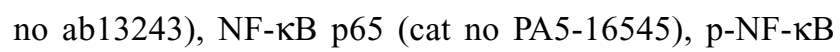
p65 (cat no PA5-37718), inhibitor of NF- $\kappa \mathrm{B}$ kinase alpha (IKK $\alpha$, cat no PA5-17803), phospho-IKK $\alpha$ (p-IKK $\alpha$, cat

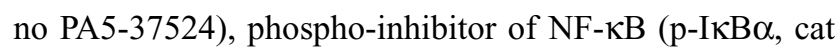
no PA5-38603), goat anti-rabbit $\operatorname{IgG}(\mathrm{H}+\mathrm{L})$ secondary antibody, biotin (cat no 31820), mitogen activated protein kinase (MAPK) isoforms: p38 (p38 MAPK, cat no AHO1202), p-p38 MAPK (cat no MA5-15182), c-JUN N-terminal kinase (JNK, cat no LF-PA0047), phospho-JNK (p-JNK, cat no PA5-37698), extracellular signal-regulated kinase (ERK, cat no LF-MA0215), phospho-ERK (p-ERK, cat no 36-8800), and $\beta$-actin (cat no ab8227). Other chemicals were of high analytical purity.

\section{Animals}

Animal handling and use were in accordance with the guidelines of the Guide for the Care and Use of Laboratory Animal of the National Institutes of Health (NIH, Bethesda, MD, USA). The experimental protocol was approved by the Research Ethics Committee, Taibah University, Saudi Ara- 
bia and Research Ethics Committee, Faculty of Pharmacy, Mansoura University, Egypt. Male Wistar rats (120-150 g) were obtained from the Animal Facility, College of Pharmacy, Taibah University. Animals were held under standard conditions of 12-hour dark/light cycle, temperature $\left(25 \pm 2^{\circ} \mathrm{C}\right)$, and humidity $(60 \%-70 \%)$. Animals were freely fed standard rat chow and water.

\section{Experimental protocol}

Rats were randomly divided into four groups of eight animals each and were treated as follows.

1. Control group where rats received oral DMSO once daily for 2 weeks.

2. DOX group: rats injected with DOX $(2.5 \mathrm{mg} / \mathrm{kg})$ six times over 2 weeks. This would result in a cumulative dose of $15 \mathrm{mg} / \mathrm{kg}$ body weight. ${ }^{18}$

3. DOX + Pris groups: in the two remaining groups, rats were treated with Pris at two different doses $(0.5,1 \mathrm{mg} /$ $\mathrm{kg}$, intraperitoneal [IP]) once daily for 1 week before and 2 weeks contaminant with DOX injection. Pris doses were selected based on previous studies that reported its potent anti-inflammatory activity. ${ }^{15}$

At the end of the treatment period, rats were weighted and anesthetized using ketamine $(75 \mathrm{mg} / \mathrm{kg}$, IP) and then subjected to electrocardiography (ECG) recording. Blood samples were obtained; serum was separated and frozen at $-20^{\circ} \mathrm{C}$ for estimation of biochemical parameters. Rats were humanely killed; their hearts were dissected and washed with PBS (10 $\mathrm{mM} \mathrm{PO}_{4}^{3-}, 137 \mathrm{mM} \mathrm{NaCl}$, and $2.7 \mathrm{mM} \mathrm{KCl} ; \mathrm{pH}=7.4$ ) and then plotted dry on filter paper and weighted. Relative heart weight was determined as the ratio of the absolute weight of the heart divided by the body weight multiplied by 100 .

Small piece of left ventricle was immediately flesh frozen and kept at $-80^{\circ} \mathrm{C}$ for measurements of gene expression. Another piece of the ventricle was homogenized in PBS and then centrifuged at $10,000 \times g$ for 20 minutes at $4^{\circ} \mathrm{C}$. The supernatant was kept at $-20^{\circ} \mathrm{C}$ till further analysis of oxidative stress parameters, antioxidants, and cytokines. The remaining portion of the left ventricle was fixed in $10 \%$ neutral buffered formaldehyde and submitted for histopathological and immunohistochemical (IHC) analysis.

\section{ECG}

Needle electrodes were inserted under the skin of the animals in lead II position. ECG was recorded and analyzed using an iWorx data recorder and the Labscribe2 software (model 214; iWorx Systems Inc., Dover, NH, USA).

\section{Serum biochemical analysis}

Serum CK-MB, LDH, cTnI, and cTnT levels were determined based on the instructions of the kits.

\section{Histopathological and IHC analysis}

Heart specimen was stained with H\&E and examined using a light microscope. Heart lesions were determined based on the degree of myofibrillar loss, apoptosis, necrosis, and fibrosis. The severity of myocardial injury was graded according to a semiquantitative score ranking from 0 to 3 depending on the degree and extent of histopathological alteration. ${ }^{13}$ Another heart specimen was stained with Masson trichrome (MT) stain for assessment of fibrosis development and deposition of ECM in the cardiac tissue. The fibrosis percentage was calculated as the ratio of the interstitial fibrosis area relative to the total specimen area. ${ }^{18}$

IHC for NF- $\mathrm{KB}$ p65 was automatically performed using Ventana BenchMark XT system (Ventana Medical Systems, Oro Valley, AZ, USA) as described previously. ${ }^{13}$ In brief, paraffin-embedded heart slides were dewaxed and rehydrated. Heat-induced antigen retrieval was achieved by boiling tissue sections in $10 \mathrm{mM}$ citrate buffer ( $\mathrm{pH}$ 6.0) for 10 minutes in microwave followed by cooling at room temperature for 20 minutes. Endogenous peroxidase activity was blocked with $3 \%$ hydrogen peroxide. Heart sections were then incubated with primary antibodies against rabbit polyclonal anti-NF- $\kappa B$ p65 (1:100) antibody at room temperature for 30 minutes. Biotin-conjugated anti-rabbit secondary antibody (1: 300) was incubated at $37^{\circ} \mathrm{C}$ for 2 hours. Diaminobenzidine was utilized for visualization of IHC reactions. Hematoxylin was used for counterstaining of sections. Semiquantitative analysis was performed using the open resource digital image analysis software (ImageJ, 1.46a; NIH), implemented with the IHC Profiler plug-in developed by Varghese et al, ${ }^{19}$ which creates a pixel-by-pixel analysis profile of a digital IHC image, and further assigns an OD score in a four tier system: 1 - negative, 2 - low positive, 3 - positive, and 4 - high positive. All images were captured at the same magnification and with the same levels of contrast and brightness.

\section{Estimation of hydroxyproline content}

The hydroxyproline content of the cardiac tissue reflects the total amount of collagen and so it was quantified to assess the degree of cardiac fibrosis. Detection was performed according to the kit procedures. Briefly, $100 \mathrm{mg}$ of the right ventricle was homogenized in $\mathrm{dH}_{2} \mathrm{O}$. Tissue homogenates were mixed with concentrated $\mathrm{NaOH}(10 \mathrm{~N})$ in a tightened screw-capped polypropylene vial and then heated at $120^{\circ} \mathrm{C}$ 
for 1 hour. The alkaline lysate was cooled on ice, neutralized, and centrifuged to obtain supernatants. The hydrolysates were dried, oxidized, and finally reacted with 4-(dimethylamino) benzaldehyde. The resultant colored product was detected at $560 \mathrm{~nm}$ and found to be proportional to the hydroxyproline present in the sample.

\section{Oxidative stress and antioxidant markers}

Levels of 4-HNE, MDA, PC, 8-OHdG, GSH, and SOD were determined in the cardiac homogenates following the protocols of the kits.

\section{Real-time PCR}

Total RNA was extracted from heart tissues using RNeasy Mini Kit according to the manufacturer's protocol (Qiagen NV, Venlo, the Netherlands). Each sample was subjected to purity quantification using OD 260/280 nm absorbance ratio. Pure samples with a 260/280 ratio between 1.8 and 2.1 were selected for subsequent experiments. cDNA was obtained from RNA $(1 \mu \mathrm{g})$ using a cDNA reverse transcription kit as described in the manufacturer's protocol (Qiagen NV). Real-time quantitative PCR (SYBR Green PCR Master Mix Kit) was used to determine the mRNA expression of targeted genes as described previously. ${ }^{17}$ Sequences of the primers are listed in Table 1. PCR efficiencies were assessed using serial dilutions of cDNA (Table S1). Specificity of PCR products was verified by melting curve analysis. $\beta$-actin was used as a normalization control. The relative gene expression was analyzed using the $\Delta \Delta \mathrm{CT}$ method.

\section{Western blot analysis}

This was conducted as previously described. ${ }^{20}$ In brief, total tissue proteins were extracted according to the instructions of the total protein extraction kit and the protein concentration was measured using the BCA Protein Assay Kit. Equal amounts of protein $(20 \mu \mathrm{g})$ were loaded into the wells of $8 \%$ SDS-PAGE gel, transferred to a cellulose acetate membrane, and then blocked with $5 \%$ nonfat milk. The membranes were incubated $\left(4^{\circ} \mathrm{C}\right)$ overnight with the primary antibodies including TGF- $\beta 1$, MMP-2, MMP-9, TIMP-1, Nrf2, GCLc, NQO1, HO-1,

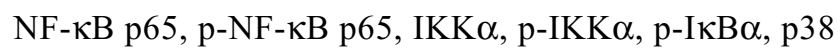
MAPK, p-p38 MAPK, JNK, p-JNK (diluted to 1:1,000), ERK (diluted to 1:2,000), and p-ERK (diluted to 1:250). The membranes were incubated for 1 hour with the secondary antibodies $(1: 200)$ at room temperature before the detection of immunoreactive proteins. $\beta$-Actin $(1: 1,000)$ was used as an internal control.

\section{ELISA assay \\ NF-kB p65 activation}

Nuclear extract of the cardiac tissue was obtained using the extraction kit. NF- $\kappa$ B p65 activation was measured based on the instruction manual of the kit. Results were represented as OD $450 \mathrm{~nm}$.

\section{Cytokines}

Levels of NOx, TNF- $\alpha$ and IL-6 were quantified in the cardiac homogenates based on the protocols provided by the manufacturer of the kits.

\section{Statistical analysis}

One-way ANOVA followed by Tukey-Kramer multiple comparison test was used for statistical comparisons between different groups. Values are presented as means \pm standard error for eight rats in each group. $P$-value $<0.05$ was considered significant.

Table I Primers used for real time-PCR measurements

\begin{tabular}{|l|l|l|l|l|}
\hline Gene & Forward primer & Reverse primer & $\begin{array}{l}\text { Product } \\
\text { size }\end{array}$ & $\begin{array}{l}\text { NCBI reference } \\
\text { sequence }\end{array}$ \\
\hline TGF- $\beta$ I & AGGGCTACCATGCCAACTTC & CCACGTAGTAGACGATGGGC & 168 & NM_02I578 \\
\hline$M M P-2$ & AGAAGGCTGTGTTCTTCGCA & AAAGGCAGCGTCTACTTGCT & 128 & NM_03I054 \\
\hline MMP-9 & GATCCCCAGAGCGTTACTCG & GTTGTGGAAACTCACACGCC & 132 & NM_03I055 \\
\hline TIMP-I & TGCTCAAAGGATTCGACGCT & AGCAGGGCTCAGATTATGCC & 200 & NM_0538I9 \\
\hline Fibronectin & GGATCCCCTCCCAGAGAAGT & GGGTGTGGAAGGGTAACCAG & 188 & NM_0I9I43 \\
\hline Coll $a l$ & GATGGACTCAACGGTCTCCC & CGGCCACCATCTTGAGACTT & 185 & NM_053304 \\
\hline Nrf2 & TTTGTAGATGACCATGAGTCGC & TGTCCTGCTGTATGCTGCTT & 142 & NM_03I789 \\
\hline GCLC & CCACTGTCCAAGGTTGACGA & CTTGCTACACCCATCCACCA & 126 & NM_0I28I5 \\
\hline GCLm & TCAAGCTCACAACTCAGGGG & CAAACCACCACATTCACGCC & 134 & NM_0I7305 \\
\hline NQOI & GGCCATCATTTGGGCAAGTC & TCCTTGTGGAACAAAGGCGA & 145 & NM_0I7000 \\
\hline HO-I & TCTGCAGGGGAGAATCTTGC & TTGGTGAGGGAAATGTGCCA & 135 & NM_012580 \\
\hline
\end{tabular}

Abbreviations: Coll $\alpha$ I, collagen type I alpha I; GCLc, glutamate-cysteine ligase catalytic subunit; GCLm, glutamate-cysteine ligase modifier; HO-I, heme-oxygenase-I; MMP-2, metalloproteinase-2; MMP-9, metalloproteinase-9; NCBI, National Center for Biotechnology Information; NQOI, NAD(P)H dehydrogenase quinone; Nrf2, nuclear erythroid 2-related factor 2; TGF- $\beta$ I, transforming growth factor-beta I; TIMP-I, tissue inhibitor of metalloproteinase-I. 


\section{Results}

\section{Pris alleviated DOX-induced cardiac damage}

Repeated DOX injections resulted in marked cardiac dysfunction in rats. ECG analysis revealed that DOX induced ECG abnormalities as there was significant increase in heart rate, QTc interval, ST segment elevation, and R wave amplitude compared to control animals (Figure 1C). Moreover, DOX administration caused a significant decrease in the relative heart weight $(P<0.001)$ compared to control animals (Figure 1B). Serum biochemical analysis showed that there was significant elevation in the indices of cardiac damage (CK-MB, LDH, cTnI, cTnT) in the DOX group compared to control group (Figure 1D).

Histopathological examination of the heart confirmed the ECG and biochemical results. Control animals exhibited normal architecture of the heart while the DOX group showed extensive deteriorated cardiac damage. Lesions occurred in the form of degeneration of cardiac muscles, coagulative necrosis, and fibrotic areas. Replacement of the cardiac tissue with fibrous tissue was well defined in the heart specimen stained with MT (Figure 2). Hydroxyproline estimation cleared the significant increase of hydroxyproline content of the heart of the DOX-treated animal compared to control indicating the increase in collagen deposition (Figure 2).

Interestingly, Pris treatment significantly reversed all of the DOX-associated cardiotoxic events in a dose-dependent manner. Pris significantly reversed DOX-induced abnormalities in the ECG and increased the relative heart weight compared to the DOX group in a dose-dependent manner. It attenuated and even normalized the elevated serum parameters of cardiotoxicity compared to the DOX group. Pathological lesions were greatly improved and fibrosis was remarkably repressed in Pris-treated animals. Furthermore, Pris attenuated DOX-induced elevation in the hydroxyproline content of the heart tissue compared to the DOX group.

\section{Pris reduced the expression of fibrogenic genes}

Repeated DOX administration caused elevation of the mRNA expression of the fibrogenic mediators TGF- $\beta 1$, MMP-2, MMP-9, fibronectin, and col1- $\alpha 1$ as compared to the control group (Figure 3). Conversely, the mRNA of TIMP-1 was significantly decreased in the DOX group. Results of Western blot were in the same direction as DOX increased the protein expression of TGF- $\beta$, MMP-2, MMP-9 contaminant with decrease in TIMP-1. Pris treatment significantly ameliorated DOX-induced alterations of the expression of these fibrogenic genes which may be correlated to the reduction of the collagen deposition in Pris-treated animals.

\section{Pris inhibited DOX-induced oxidative stress}

As shown in Figure 4, repeated DOX injection induced elevation of lipid peroxidation markers, 4-HNE, MDA, PC, and 8-OHdG, compared to the control group. In addition, DOX caused a significant reduction in GSH content and SOD activity compared to the control group. Pris treatment significantly inhibited the elevation of these lipid per-oxidative parameters simultaneously with the remarkable elevation of GSH and SOD compared to the DOX group.

\section{Pris activated Nrf2 signaling pathway}

$\mathrm{Nrf} 2$ is an important upstream regulator for antioxidant response element-driven expression of antioxidant enzymes, such as GCL, NQO1, and HO-1. As presented in Figure 5, DOX chronic administration resulted in downregulation of mRNA expression of Nrf2 as well as Nrf2 target gene (GCLc, glutamate-cysteine ligase modifier, NQO1, and HO-1) compared to control animals. Consistent with mRNA results, Western blot analysis revealed significant reduction of the protein expression of Nrf2, GCL, NQO1, and HO-1 in the DOX group. Additionally, the level of HO-1 was significantly decreased in the hearts of DOX-treated animals. On the contrary, Pris treatment significantly upregulated the mRNA and protein expression of $\mathrm{Nrf} 2$ and its target gene. Pris caused significant elevation of HO-1 level compared to the DOX group.

\section{Pris inhibited activation of MAPK/NF- $\mathrm{KB}$ signaling pathways}

To assess the mechanisms of the anti-inflammatory activity of Pris, we examined MAPK and NF- $\kappa B$ signaling pathways. Repeated DOX administration resulted in the activation of MAPK as noticed in the Western blot analysis. The phosphorylation levels of the MAPKs isoforms (p38, JNK, ERK) were elevated in the DOX-treated group although there was no change in the total protein of these isoforms. Meanwhile, Pris treatment dramatically decreased the phosphorylation of MAPKs compared to the DOX group (Figure 6A-C).

Concerning NF- $\kappa \mathrm{B}$ signaling pathway, it was greatly activated in the case of repeated DOX administration. IHC and ELISA detection of p65 (the activated subunit of NF- $\mathrm{kB}$ ) revealed the marked elevation of NF- $\mathrm{KB}$ p65 compared to control animals (Figure 6D and E). Western blot analysis showed the marked elevation of the levels of phosphorylated

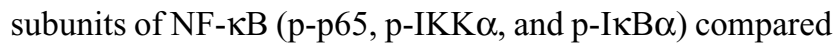


A

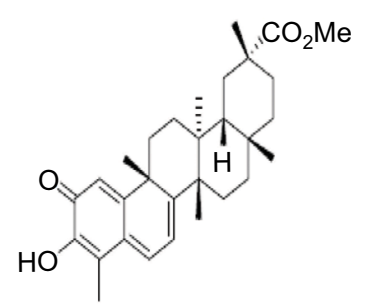

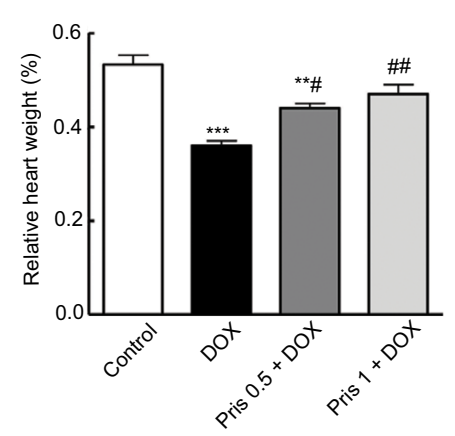

C
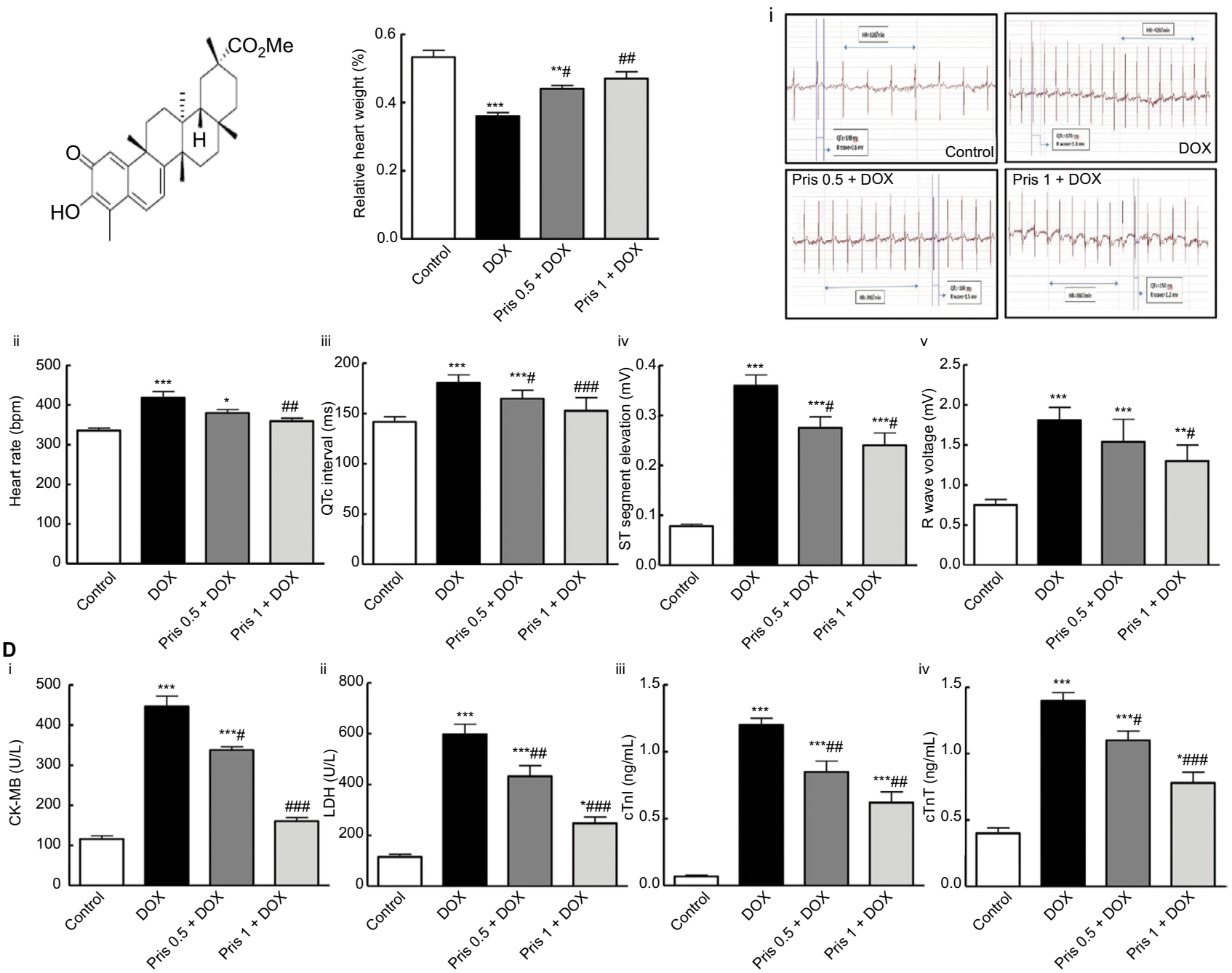

Figure I Impact of Pris on DOX-induced cardiac toxicity.

Notes: (A) Chemical structure of Pris. (B) Relative heart weight. (C) ECG variables: (i) ECG recordings; (ii) heart rate; (iii) QTc interval; (iv) ST segment elevation; (v) R wave amplitude. (D) Serum indices of cardiac damage. (i) CK-MB; (ii) LDH; (iii) cTnl; (iv) cTnT. Rats were injected with DOX (2.5 mg/kg) six times over 2 weeks. Pris was administered $(0.5, I \mathrm{mg} / \mathrm{kg}$, intraperitoneal) once daily for I week before and 2 weeks contaminant with $D O X$ injection. Data are means \pm standard error $(\mathrm{n}=8)$. $* P<0.05$, $* * P<0.0$ I, ${ }^{* * *} P<0.001$ vs the control; ${ }^{*} P<0.05,{ }^{\# P} P<0.01, \ldots+1 \times 0.001$ vs the DOX group (one-way ANOVA).

Abbreviations: CK-MB, creatine kinase-MB; cTnl, cardiac troponin I; cTnT, cardiac troponin T; DOX, doxorubicin; ECG, electrocardiography; LDH, lactate dehydrogenase; Pris, pristimerin.

to the control group indicating the activation of NF- $\kappa \mathrm{B}$. Conversely, Pris treatment effectively counteracted the activation of NF- $\kappa \mathrm{B}$ and suppressed the phosphorylation of $\mathrm{p} 65$, IKK $\alpha$, and $\mathrm{I} \kappa \mathrm{B} \alpha$ (Figure 6F).

Activation of NF- $\kappa \mathrm{B}$ is closely associated with the enhancement of inflammatory cytokines release. This was clearly evident through the elevation of NOx, TNF- $\alpha$, and IL-6 levels. However, NF- $\kappa \mathrm{B}$ signaling upswing was effectively depressed simultaneously with reduction of the levels of NOx, TNF- $\alpha$, and IL-6 in the case of Pris treatment (Figure 6G).

\section{Discussion}

DOX-induced cardiotoxicity remains a major clinical challenge to its ubiquitous therapeutic use in oncology. ${ }^{4}$ Hence, new safe chemo-protective agents that could prevent the detrimental effects of DOX need to be found. Results of the present study provide new evidences of the cardioprotective activity of Pris against DOX-induced cardiomyopathy and fibrosis which may be mediated via Nrf2 activation and MAPK/NF- $\kappa$ B inhibition.

DOX-induced cardiotoxicity may occur in the form of acute or chronic cardiomyopathy. Acute cardiotoxic effects of DOX occur after administration of a high dose of DOX while chronic cardiotoxicity results from repeated DOX administration and it is dose dependent. Cardiac fibrosis is noted in chronic DOX administration and it contributes to cardiac stiffness and dysfunction. Fibroblasts overproduce collagen which replaces the necrotic or apoptotic myocytes..$^{3,21,22}$ The 
A
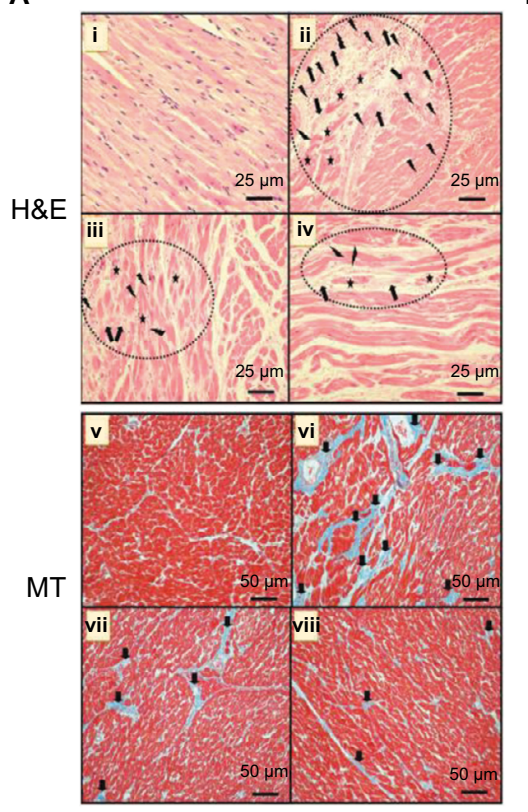

B

\begin{tabular}{l|c|c|c|c}
\hline \hline Histopathological changes & Control & DOX & $\begin{array}{c}\text { Pris 0.5+ } \\
\text { DOX }\end{array}$ & $\begin{array}{c}\text { Pris 1+ } \\
\text { DOX }\end{array}$ \\
\hline $\begin{array}{l}\text { Coagulative necrosis of cardiac muscles } \\
\text { (fragmentation and resportion with loss of } \\
\text { striation bands and nuclei) }\end{array}$ & - & +++ & ++ & + \\
\hline $\begin{array}{l}\text { Granulation tissue (new blood capillaries and } \\
\text { fibroblast infiltration) }\end{array}$ & - & +++ & ++ & + \\
\hline \begin{tabular}{l} 
Collagen deposition and fibrosis \\
\hline
\end{tabular}
\end{tabular}

C

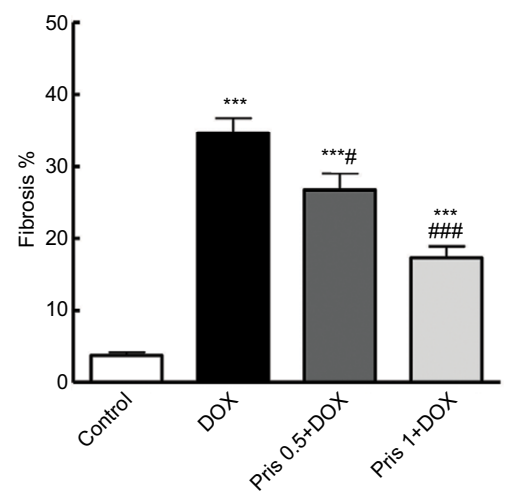

D

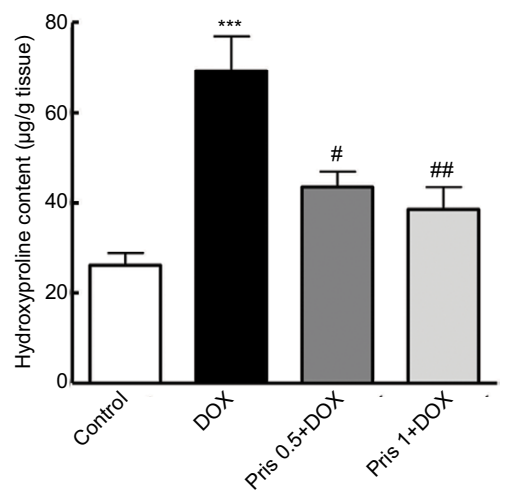

Figure 2 Impact of Pris on DOX-induced cardiac histopathological damage and alteration in hydroxyproline content.

Notes: (A) Heart specimen stained by H\&E stain where control rats (i) showed normal heart histology while heart specimen of DOX group (ii) showed large areas of coagulative necrosis (circle) (cardiac muscle fragmentation and resorption with loss of striation bands and nuclei) (stars). Pris treated groups (iii) and (iv) showed remarkable attenuation of DOX-induced cardiac damage. There is replacement of injured cardiac muscles with granulation tissue with new vascularization (lightning bolt), collagen deposition which matures to fibrous tissue (arrows) $\times 400$; scale bar $25 \mu \mathrm{m}$. Heart specimen stained by MT stain where control rats ( $\mathrm{v}$ ) showed no signs of fibrosis, while DOX group (vi) cardiac muscles showed replacement of injured cardiac muscles, with blue stain indicating increased collagen and fibrous tissue (small arrows) $\times 200$; scale bar $50 \mu \mathrm{m}$. Rats pretreated with Pris (vii, viii) showed dose-dependent amelioration of DOX-induced cardiac lesions and fibrosis. (B) Semiquantitative analysis of the severity of histopathological changes in cardiac muscle fibers stained with H\&E. (C) Fibrosis percentage in heart muscles in specimen stained with MT. (D) Hydroxyproline content in cardiac tissue. Rats were injected with DOX $(2.5 \mathrm{mg} / \mathrm{kg})$ six times over 2 weeks. Pris was administered $(0.5$, I mg/kg, intraperitoneal) once daily for I week before and 2 weeks contaminant with DOX injection. Data are means \pm standard error $(n=8)$. ${ }^{* * *} P<0.00$ I vs the control; ${ }^{\#} P<0.05$, ${ }^{\# P} P<0.0$ I, ${ }^{\# \# P} P<0.00$ I vs the $D O X$ group (one-way ANOVA).

Abbreviations: DOX, doxorubicin; MT, Masson's trichrome; Pris, pristimerin.

present study revealed that repeated DOX administration produced profound cardiac damage and dysfunction. DOXassociated depression of the cardiac function was evident through ECG abnormalities. Similar results were reported in previous studies. ${ }^{22}$ In addition, there was an increase in the serum biomarkers of myocardial toxicity, namely CK-MB, LDH, cTnI, and cTnT, indicating loss of cell membrane integrity of myocytes. These ECG and biochemical data were supported by the results of the histopathological examination of the heart specimen which revealed severe cardiac lesions and remarkable fibrosis, that was clearly noticed in the heart specimen stained by MT stain. Collagen deposition and development of cardiac fibrosis were further confirmed by the significant increase in the hydroxyproline content which is the main constituent of collagen. These findings agree with the outcomes of previous studies. ${ }^{18,23-26}$ Interestingly, Pris ameliorated all DOX-induced toxic events as presented by the amelioration of ECG abnormalities, lowering of cardiotoxic biomarkers, and improvement of the heart lesions and fibrosis. These data indicate that Pris enabled marked preservation of the myocardium integrity and restoration of the cardiac function.

MMPs are a family of zinc-dependent proteases that play a significant role in the degradation of fibrillar ECM proteins and maintain normal tissue growth and remodeling. There is a balance between MMPs and their TIMPs which is altered during myocardial injury and inflammation resulting in the deregulation of ECM turnover and myocardial fibrosis. ${ }^{27}$ Previous studies have linked the rise of MMPs expression with the progression of DOX-induced cardiac dysfunction $^{3,28}$ and fibrosis. ${ }^{22}$ In line with the previous context, our results showed the elevation of the expression of MMP-2 and MMP-9 in the heart of DOX-treated animals simultaneously with depressed expression of TIMP-1. Conversely, Pris markedly downregulated the expression of MMP-2 and MMP-9 contaminant with upregulation of TIMP-1. These results suggest that Pris could effectively modulate the principal regulators of ECM homeostasis which may explain in part Pris-induced amelioration of cardiac fibrosis in DOX-treated animals. 
A
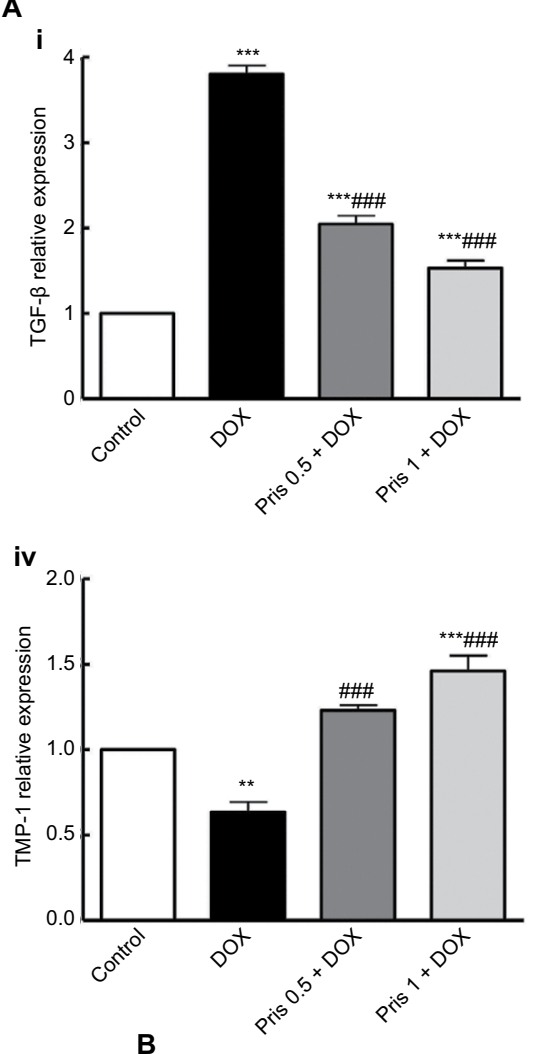

B

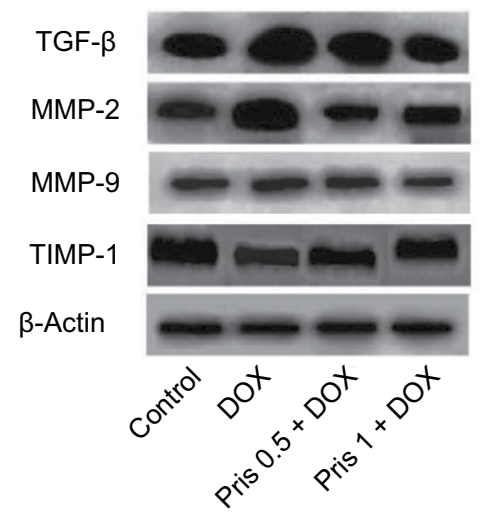

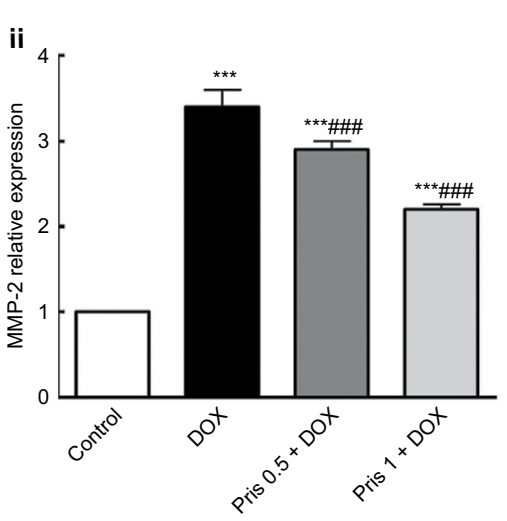
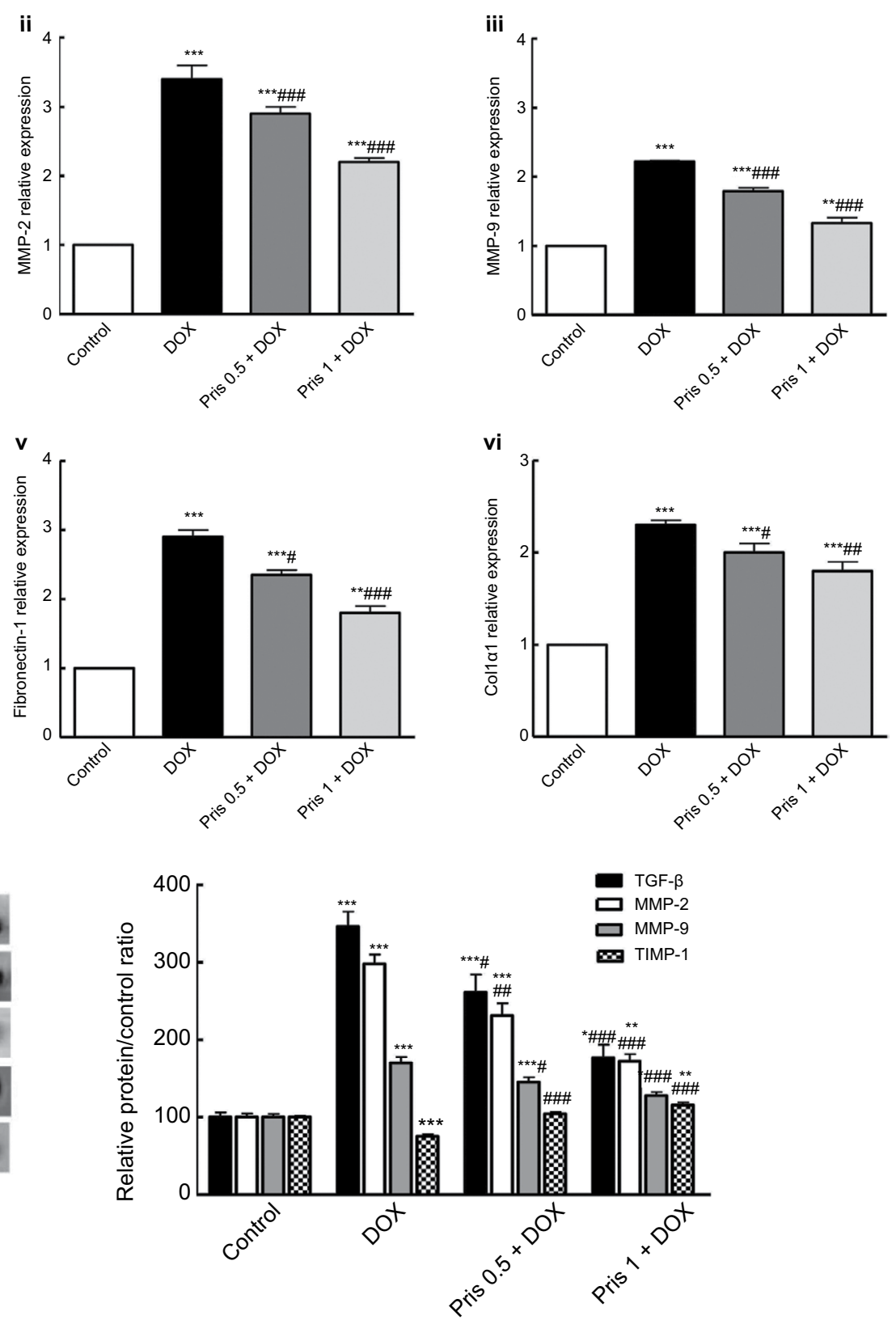

Figure 3 Effect of Pris on DOX-induced change of mRNA and protein expression of fibrogenic genes.

Notes: (A) mRNA expression of (i) TGF- $\beta$, (ii) MMP-2, (iii) MMP-9, (iv) TIMP-I, (v) fibronectin-I, and (vi) Coll $\alpha$ I in cardiac tissue. (B) Western blot for TGF- $\beta$, MMP-2, MMP-9, TIMP-I, and their relative protein quantification. $\beta$-Actin served as an internal control. Rats were injected with DOX (2.5 mg/kg) six times over 2 weeks. Pris was administered $\left(0.5, I \mathrm{mg} / \mathrm{kg}\right.$, intraperitoneal) once daily for I week before and 2 weeks contaminant with $D O X$ injection. Data are the mean $\pm s t a n d a r d$ error $(\mathrm{n}=8)$. ${ }^{*}<<0.05$, ${ }^{* * P} P<0.01$, ${ }^{* * *} P<0.001$ vs the control; ${ }^{*} P<0.05,{ }^{\# P} P<0.01, \ldots P<0.001$ vs the DOX group (one-way ANOVA).

Abbreviations: Coll $\alpha$ I, collagen type I alpha I; DOX, doxorubicin; MMP, metalloproteinase; Pris, pristimerin; TGF- $\beta$, transforming growth factor- $\beta$; TIMP-I, tissue inhibitor of metalloproteinase-I.

The pathogenesis of fibrosis includes several mechanistic pathways. The inflammatory and growth factor signaling pathways mediated by TGF- $\beta$ are the basic pathways for fibrosis. TGF- $\beta 1$ is secreted by cardiac myofibroblasts and controls the progression of cardiac fibrosis. DOX-induced cardiac fibrosis is associated with the overexpression of TGF- $\beta 1{ }^{18,26}$ Our results confirmed these previous findings as DOX administration caused elevation of the pro-fibrogenic markers TGF- $\beta$, fibronectin, and Col1 $\alpha 1$. On the other hand, we observed that Pris-treated groups exhibited attenuation of these pro-fibrogenic genes leading to depression of fibrosis development. Taken together, these data provide evidence that Pris could effectively attenuate fibrosis development in DOX-treated animals via modulation of fibrogenic genes.

ROS produce direct tissue injury and induce inflammatory responses via the activation of different pro-inflammatory cytokines..$^{29}$ Increased oxidative stress and the resultant lipid peroxidation cause tissue necrosis and inflammation 

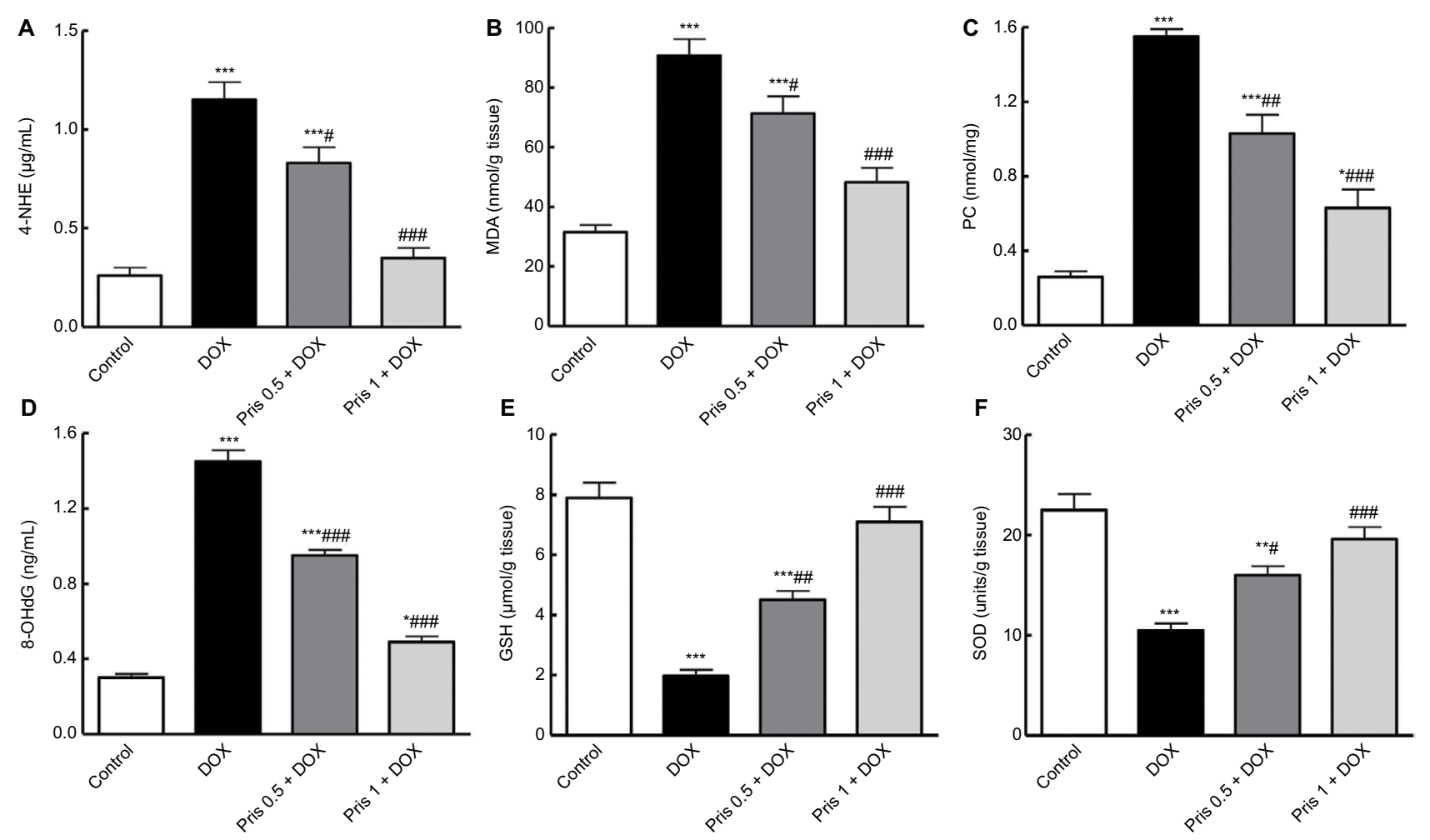

Figure 4 Impact of Pris on DOX-induced oxidative stress and depression of antioxidant parameters in cardiac tissue.

Notes: (A) 4-HNE; (B) MDA; (C) PC; (D) 8-OHdG; (E) reduced GSH; (F) SOD. Rats were injected with DOX (2.5 mg/kg) six times over 2 weeks. Pris was administered $(0.5,1 \mathrm{mg} / \mathrm{kg}$, intraperitoneal) once daily for I week before and 2 weeks contaminant with $D O X$ injection. Data are means $\pm s t a n d a r d$ error $(n=8)$. $* P<0.05$, $* * P<0.01$, ${ }^{*} * * P<0.001$ vs the control; ${ }^{\#} P<0.05,{ }^{\# P} P<0.01,{ }^{\#} P<0.00$ I vs the DOX group (one-way ANOVA).

Abbreviations: 4-HNE, 4-hydroxynonenal; 8-OHdG, 8-hydroxy-2-deoxyguanosine; DOX, doxorubicin; GSH, glutathione; MDA, malondialdehyde; PC, protein carbonyl; Pris, pristimerin; SOD, superoxide dismutase.

and hence promote the progression of tissue fibrogenesis. ${ }^{30}$ As the myocardium has a restricted regenerative capacity, and also is characterized by its relative lack of endogenous antioxidants, this makes the cardiac tissue more vulnerable to oxidative damage. ${ }^{2}$ Overproduction of ROS extensively harms myocardial tissue and destructs cell membrane integrity causing leakage of cardiac diagnostic markers. ${ }^{31}$ Previous investigations have shown the basic role of ROS and oxidative stress in the mediation of DOX-induced cardiotoxicity. ${ }^{4,32-34}$ DOX is known to generate extensively ROS such as hydroxyl radicals and hydrogen peroxide that induce lipid peroxidation and accumulation of the highly reactive electrophile, 4-HNE, which is a good biomarker of DOXinduced oxidative stress. ${ }^{35}$ Furthermore, DOX administration has been shown to depress the level of GSH in the heart. ${ }^{36}$ Antioxidant treatment has shown its ability to attenuate the progression of DOX cardiotoxicity and to improve cardiac function. ${ }^{37}$ In the present study, DOX-induced deleterious oxidative stress was evident through the elevation of PC (marker of the protein oxidative injury), 4-HNE (marker of lipid peroxidation), and 8-OHdG (marker of oxidative DNA injury). However, these indices were significantly depressed in the Pris-treated groups. Additionally, DOX attenuated SOD activity and caused a depletion of myocardial GSH which were reversed by Pris treatment. Therefore, these data indicate the potent antioxidant activity of Pris which impeded DOX-induced oxidative injury. This hypothesis is reinforced by the data of an earlier report of Hui et al, ${ }^{38}$ which demonstrated the free radical scavenging capacity of Pris in LPS-induced tissue injury.

Nrf2 is a redox-sensitive transcription factor that controls detoxification gene activity and cellular responses toward oxidative and electrophilic stress. Normally, Nrf2 is repressed in the cytosol by Kelch-like ECH-associated protein 1 (Keap1). In response to oxidative or electrophilic stress, Nrf2 is released from Keap1 and translocated into the nucleus where it induces the expression of several antioxidant genes such as GCL, NQO1, and HO-1. ${ }^{7}$ Previously, it was reported that DOX administration reduces active nuclear Nrf2 in the heart. The inhibition of Nrf2 pathway has been 
A
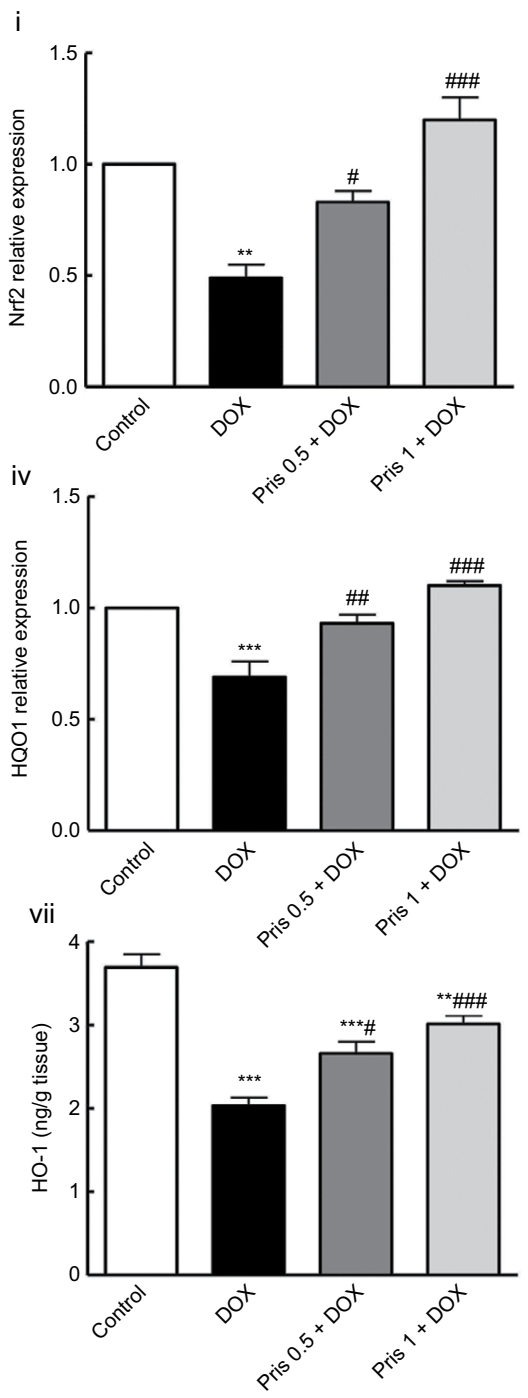

ii

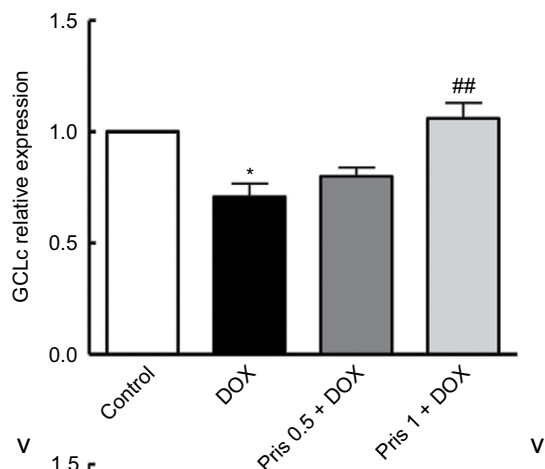

iii

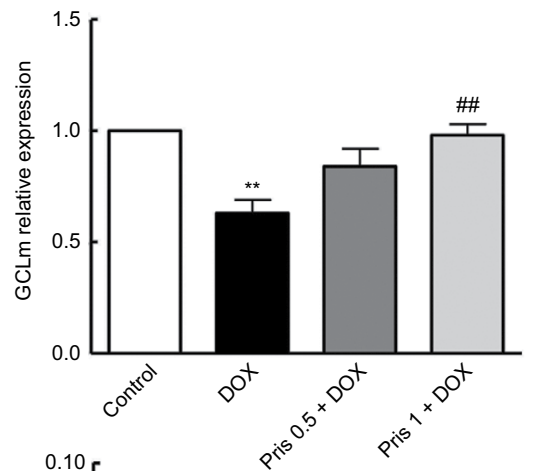

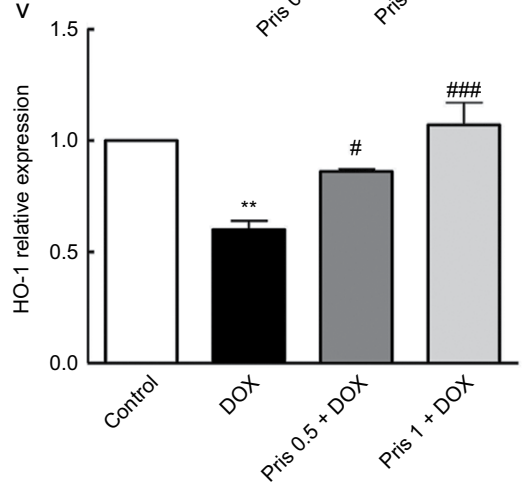

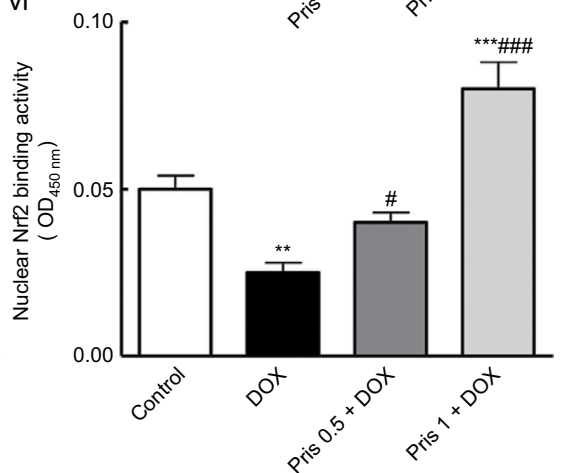

B
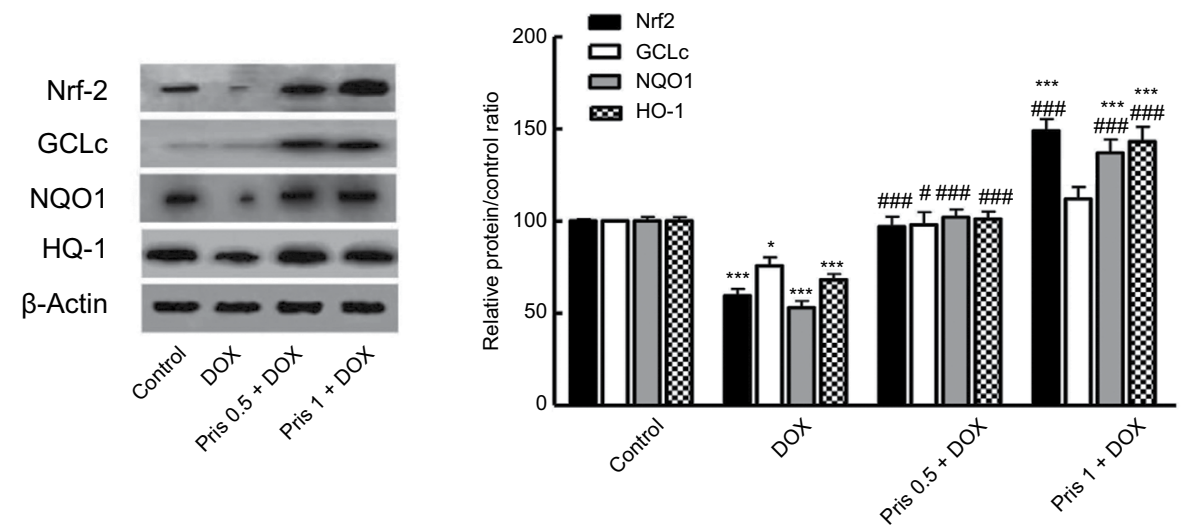

Figure 5 Effect of Pris on DOX-induced alteration of Nrf2 signaling pathway.

Notes: (A) (i-v) mRNA expression of Nrf2, GCLc, GCLm, NQOI, and HO-I. (vi) Nrf2 binding capacity. (vii) HO-I level in the heart. (B) Western blot for Nrf2, GCLc, $\mathrm{NQOI}, \mathrm{HO}-\mathrm{I}$, and their relative protein quantification. $\beta$-Actin served as an internal control. Rats were injected with DOX (2.5 mg/kg) six times over 2 weeks. Pris was administered $(0.5,1 \mathrm{mg} / \mathrm{kg}$, intraperitoneal) once daily for I week before and 2 weeks contaminant with $D O X$ injection. Data are the mean $\pm s t a n d a r d$ error $(n=8)$. $* P<0.05$, $* * P<0.0$ I, ${ }^{* * * P}<0.00$ I vs the control; ${ }^{*} P<0.05,{ }^{\# P} P<0.01,{ }^{\#} P<0.001$ vs the DOX group (one-way ANOVA).

Abbreviations: DOX, doxorubicin; GCLc, glutamate-cysteine ligase catalytic subunit; GCLm, glutamate-cysteine ligase modifier; HO-I, heme-oxygenase-I; NQOI, NAD(P) $\mathrm{H}$ dehydrogenase quinone; Nrf2, nuclear erythroid 2-related factor 2; Pris, pristimerin. 
A

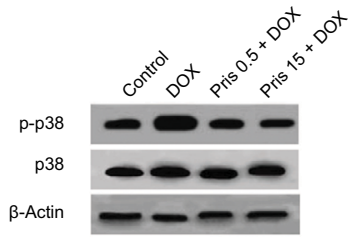

B

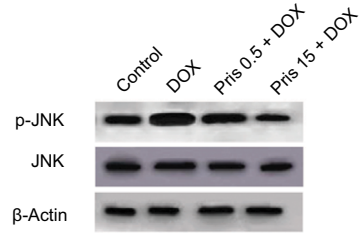

c

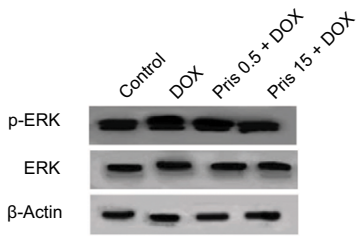

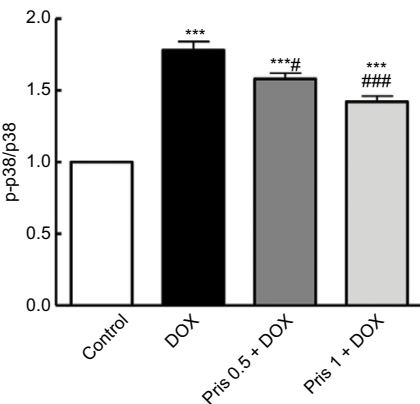

D

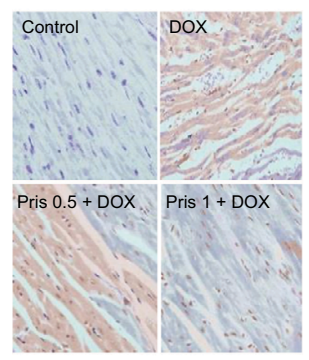

$\mathbf{F}$

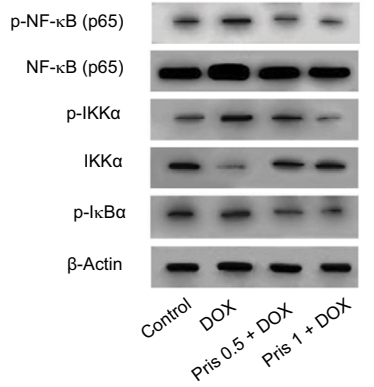

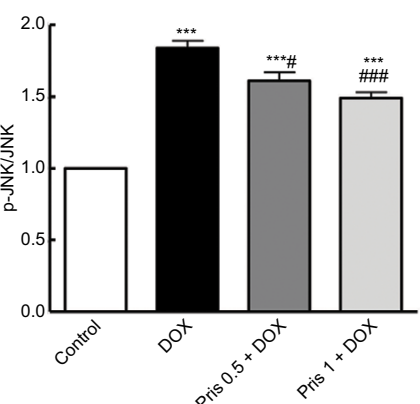

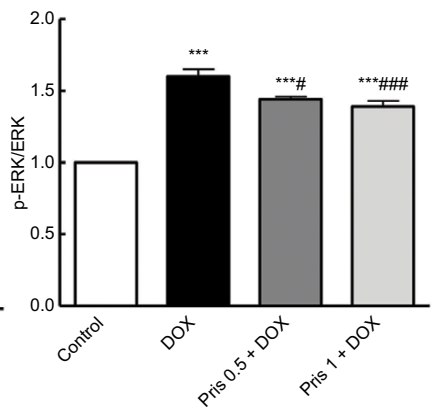

E
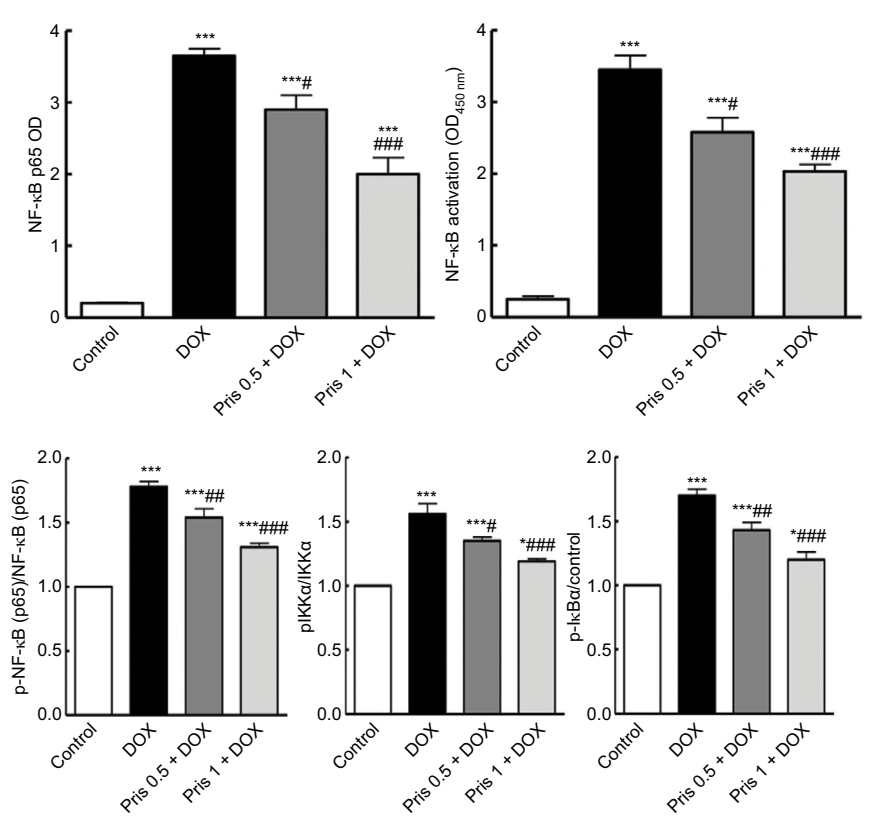

G

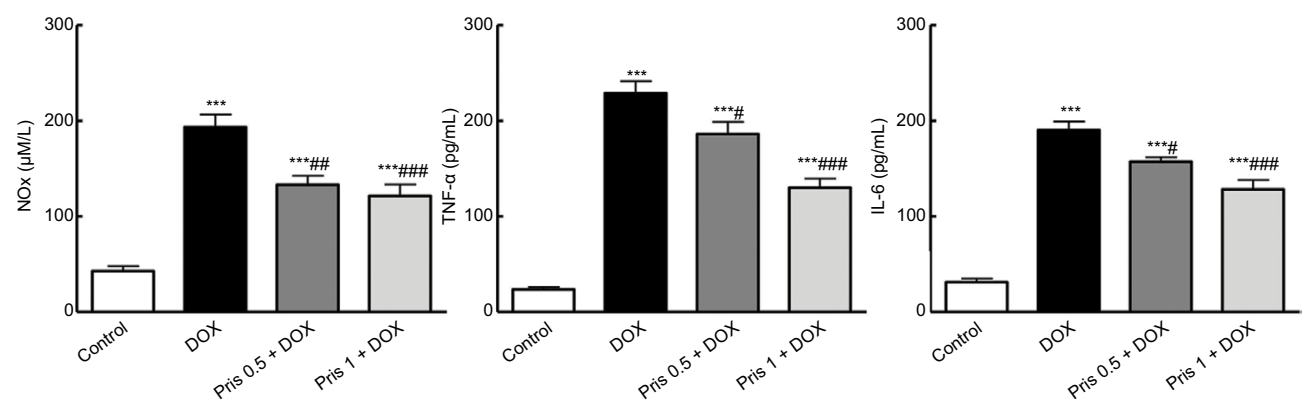

Figure 6 Effect of Pris on DOX-induced alteration of MAPK/NF- $K B$ signaling pathways.

Notes: Western blot showing the protein expression of (A) p-p38 and p38, (B) p-JNK and JNK, (C) p-ERK and ERK, and quantification of the protein intensities. $\beta$-Actin was used as an internal control. (D) Specimen of the cardiac tissue in different groups showing different protein expressions of NF-KB p65 via IHC staining and semiquantitative analysis of IHC expressed as OD across 10 different fields for each section; $\times 400$, scale bar $25 \mu \mathrm{m}$. (E) NF- $\mathrm{KB}$ p 65 activation in the cardiac tissue. (F) Western blot for NF$\kappa B$ p65, p-NF- $K B$ p 65, IKK $\alpha$, phospho-IKK $\alpha$, p-IKB $\alpha$, and quantification of the protein intensities. $\beta$-Actin was used as an internal control. (G) Cytokine levels in the cardiac tissue. Rats were injected with DOX (2.5 mg/kg) six times over 2 weeks. Pris was administered $(0.5,1 \mathrm{mg} / \mathrm{kg}$, intraperitoneal) once daily for I week before and 2 weeks contaminant with DOX injection. Data are the mean \pm standard error $(\mathrm{n}=8)$. ${ }^{*} P<0.05,{ }^{*} * * P<0.00$ I vs the control; ${ }^{*} P<0.05,{ }^{\# P} P<0.01$, ${ }^{\# \# P} P<0.001$ vs the DOX group (one-way ANOVA).

Abbreviations: DOX, doxorubicin; ERK, extracellular signal-regulated kinase; IHC, immunohistochemical; IKK $\alpha$, inhibitor of nuclear factor kappa-B kinase alpha; JNK, c-JUN N-terminal kinase; MAPK, mitogen activated protein kinase; NF-kB, nuclear factor kappa-B; p-ERK, phospho-ERK; p-lkB $\alpha$, phospho-inhibitor of NF-kBp-JNK, phosphoJNK; Pris, pristimerin. 
linked to suppression of the protective enzymes and so failure of the heart to protect itself against DOX-induced oxidative and electrophilic damage. ${ }^{36}$ Upregulation of Nrf2 and induction of the cyto-protective antioxidant target genes conferred protection against acute cardiotoxic effects of DOX. ${ }^{8}$ In agreement with this, our results showed that DOX induced a decrease of the expression and activity of Nrf2 contaminant with depression of its target genes. The depression of Nrf2-targeted genes could be responsible in part in the depletion of GSH. ${ }^{7}$ In contrast, co-administration of Pris enhanced the expression and activity of Nrf2 and its target genes. These data lead us to the presumption that the cardioprotective activity of Pris may be partially mediated via activation of Nrf2 pathway which enhances the antioxidant capacity of the heart.

MAPKs families are serine/threonine protein kinases that control signal transduction involved in cell proliferation, differentiation, survival, and death in addition to their fundamental role in the regulation of inflammatory mediators. MAPK signaling pathways involve three kinase isoforms: p38, ERK, and JNK. Several lines of evidence indicate that ROS activate both JNK and p38 MAPK leading to the enhancement of apoptosis. ${ }^{39}$ Previous investigations have demonstrated the participation of MAPKs in the pathophysiology of DOX-induced cardiac injury. ${ }^{40-42}$ Our results were similar and confirmed the role of MAPK in the mediation of DOX-induced cardiotoxic effects. On the contrary, Pris supplementation suppressed DOX-induced MAPKs activation and so our results indicated that the cardioprotective effect of Pris might involve the modulation of MAPK signaling pathway. These findings are in agreement with those of Jin et $\mathrm{al}^{43}{ }^{43}$ who reported the inhibitory effect of Pris on MAPK signaling pathway in case of ovalbumin-induced allergic airway inflammation.

$\mathrm{NF}-\kappa \mathrm{B}$ signaling is another major signal transduction pathway that is activated in response to oxidative stress and has crucial rules in cell survival, inflammation, and immune responses. This ubiquitous transcription factor controls the induction of various inflammatory cytokines. Normally, NF- $\mathrm{B}$ p 65 subunit binds to its inhibitory counterpart $\mathrm{I} \kappa \mathrm{B} \alpha$ and other $\mathrm{I} \kappa \mathrm{B}$ proteins to form an inactive complex that is present in the cytoplasm. Upon activation, $\mathrm{I} \kappa \mathrm{B} \alpha$ is phosphorylated leading to activation and translocation of NF- $\mathrm{KB}$ p65 into the nucleus where it mediates the expression of inflammatory genes. Several studies have focused on the fundamental role of NF- $\kappa$ B activation with its subsequent inflammatory cascade in the mediation of
DOX cardiotoxic effects. ${ }^{10-12,25}$ Correspondingly, elevations of TNF- $\alpha$ and IL- 6 levels are considered as indicators of the inflammatory response in case of DOX intoxication. High expression of these inflammatory cytokines has been reported during DOX administration. ${ }^{44}$ Additionally, $N F-\kappa B$ activation has been shown to enhance the generation of NOx through the induction of expression of iNOS. NOx can generate peroxynitrite in cardiomyocyte which causes cell death and exaggerated cardiac damage. Peroxynitrite is also involved with other ROS in the inhibition of multiple mitochondrial enzymes leading to the enhancement of oxidative/nitrosative stress, persistent even in the absence of DOX in the circulation/myocardium. ${ }^{45-47}$ The strong correlation between the generated ROS and NF- $\kappa \mathrm{B}$ activation results in the perseverance and exaggeration of their injurious impacts. ${ }^{48}$ Results of the present study confirmed the phosphorylation and activation of NF- $\kappa B$ signaling during DOX administration. Furthermore, the levels of the cytokines, NOx, TNF- $\alpha$, and IL-6, were significantly increased. Notably, Pris remarkably inhibited the phosphorylation of NF- $\kappa \mathrm{B}$ p 65 , IKK $\alpha$, and $\mathrm{I} \kappa \mathrm{B} \alpha$ in the cardiac tissue indicating that $N F-\kappa B$ activation was greatly depressed in Pris-treated animals. Hence, levels of the related downstream inflammatory cytokines, NOx, TNF- $\alpha$, and IL- 6 were dramatically decreased. These data are consistent with previous studies showing the inhibitory activity of Pris on NF- $\kappa \mathrm{B}$ activation and subsequent release of inflammatory cytokines. ${ }^{17,43}$ The ability of Pris to inhibit DOX-induced NF- $\kappa \mathrm{B}$ activation partially explains the underlying cardioprotective mechanism of Pris in DOX-induced cardiotoxicity. Collectively, it is acceptable to presume that Pris could repress DOX-induced cardiotoxicity via suppression of MAPK/NF- $\mathrm{B}$ phosphorylation.

\section{Conclusion}

The present findings indicate the apparent protective effects of Pris against DOX-induced cardiotoxic damage. Pris showed potent antioxidant and anti-inflammatory activities which effectively suppressed DOX-induced oxidative damage and inflammatory responses, most likely due to activation of Nrf2 and inhibition of the transcriptional activity of MAPK/ NF-кB signaling (Figure 7). Thus, it may be acceptable to suggest that the combined administration of Pris with DOX may provide a safer therapeutic strategy with a more improved therapeutic ratio of DOX. However, the cardioprotective effects of Pris against the cardiotoxic effect of DOX are worthy of further exploration. 


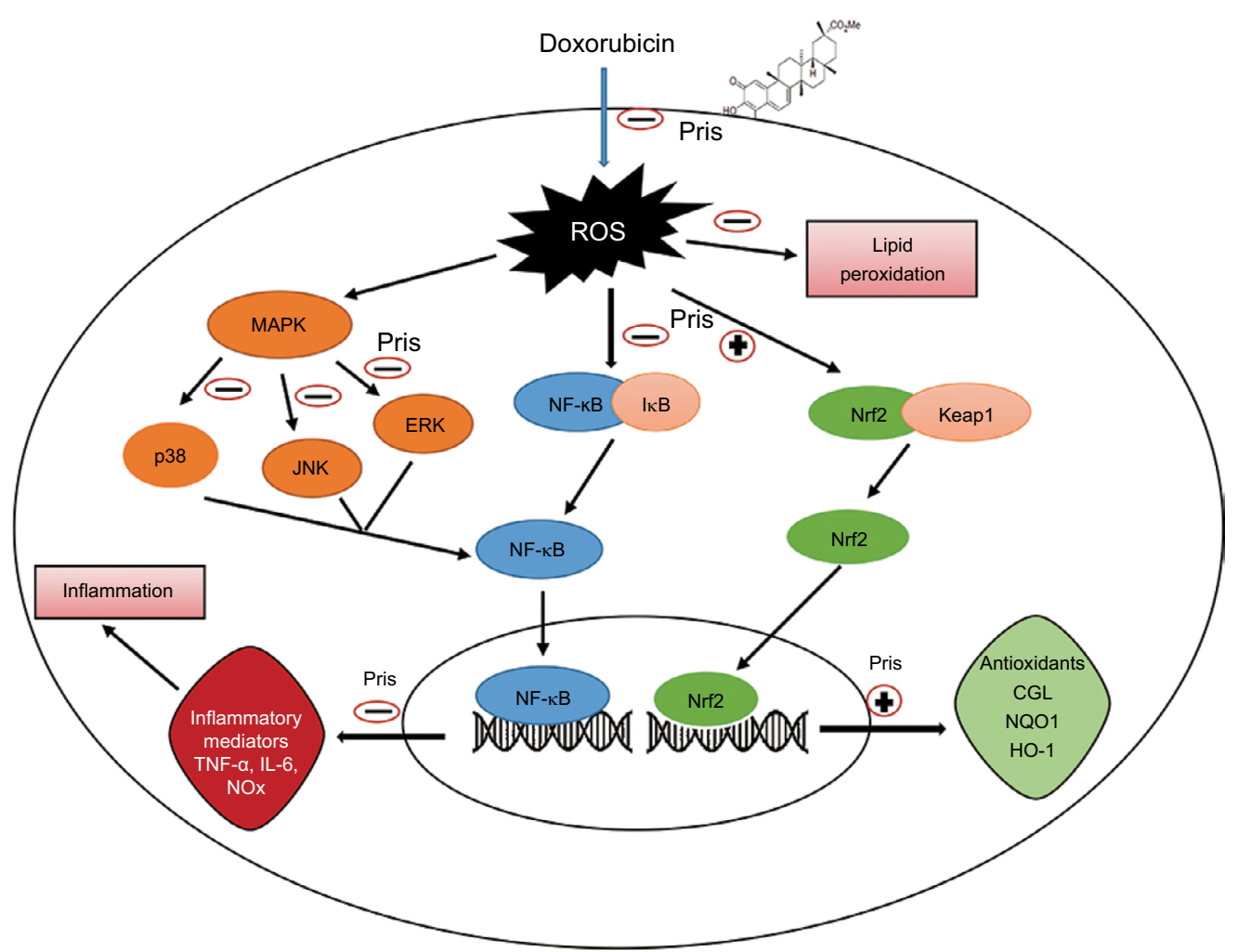

Figure 7 Schematic diagram summarizing the possible cardioprotective mechanisms of Pris against DOX-induced cardiotoxicity.

Abbreviations: ERK, extracellular signal-regulated kinase; GCL, glutamate-cysteine ligase; IKB, inhibitor of kappa B; HO-I, heme-oxygenase-I; IL-6, interleukin-6; JNK, c-JUN N-terminal kinase; KeapI, Kelch-like ECH associating protein I; MAPK, mitogen activated protein kinase; NF-kB, nuclear factor kappa-B; Pris, pristimerin; DOX, doxorubicin; NQOI, NAD(P)H dehydrogenase quinone; NOx, nitric oxide; Nrf2, nuclear erythroid 2-related factor 2; TNF- $\alpha$, tumor necrosis factor- $\alpha$.

\section{Author contributions}

Study design and conception: DS El-Agamy; experimental work: N Ahmed, DS El-Agamy, and AA Shaaban; drafting the manuscript: DS El-Agamy and HM Abo-Haded; ECG recording and analysis: HM Abo-Haded; supplementation of reagents/materials/analysis tools: KM El-Harbi, S Khoshhal, HM Abo-Haded, and MA Elkablawy; genetic assessments: KM El-Harbi and S Khoshhal; histopathology and IHC: MA Elkablawy. The authors equally contributed to the acquisition of data and manuscript revision. All authors contributed to data analysis, drafting and revising the article, gave final approval of the version to be published, and agree to be accountable for all aspects of the work.

\section{Disclosure}

The authors report no conflicts of interest in this work.

\section{References}

1. Thorn CF, Oshiro C, Marsh S, et al. Doxorubicin pathways: pharmacodynamics and adverse effects. Pharmacogenet Genomics. 2011;21(7):440-446

2. Carvalho FS, Burgeiro A, Garcia R, Moreno AJ, Carvalho RA, Oliveira PJ. Doxorubicin-induced cardiotoxicity: from bioenergetic failure and cell death to cardiomyopathy. Med Res Rev. 2014;34(1):106-135.
3. Ivanová M, Dovinová I, Okruhlicová L, et al. Chronic cardiotoxicity of doxorubicin involves activation of myocardial and circulating matrix metalloproteinases in rats. Acta Pharmacol Sin. 2012;33(4): 459-469.

4. Octavia Y, Tocchetti CG, Gabrielson KL, Janssens S, Crijns HJ, Moens AL. Doxorubicin-induced cardiomyopathy: from molecular mechanisms to therapeutic strategies. J Mol Cell Cardiol. 2012;52(6): 1213-1225.

5. Schunke KJ, Coyle L, Merrill GF, Denhardt DT. Acetaminophen attenuates doxorubicin-induced cardiac fibrosis via osteopontin and GATA4 regulation: reduction of oxidant levels. $J$ Cell Physiol. 2013;228(10):2006-2014.

6. Liu G, Liu Y, Wang R, et al. Spironolactone attenuates doxorubicin-induced cardiotoxicity in rats. Cardiovasc Ther. 2016;34(4) 216-224.

7. Ma Q. Role of nrf2 in oxidative stress and toxicity. Annu Rev Pharmacol Toxicol. 2013;53:401-426.

8. Wang LF, Su SW, Wang L, et al. Tert-butylhydroquinone ameliorates doxorubicin-induced cardiotoxicity by activating Nrf2 and inducing the expression of its target genes. Am J Transl Res. 2015;7(10):1724-1735.

9. Hajra S, Basu A, Singha Roy S, Patra AR, Bhattacharya S. Attenuation of doxorubicin-induced cardiotoxicity and genotoxicity by an indole-based natural compound 3,3'-diindolylmethane (DIM) through activation of Nrf2/ARE signaling pathways and inhibiting apoptosis. Free Radic Res. 2017;51(9-10):812-827.

10. Nozaki N, Shishido T, Takeishi Y, Kubota I. Modulation of doxorubicininduced cardiac dysfunction in toll-like receptor-2-knockout mice Circulation. 2004;110(18):2869-2874.

11. Fouad AA, Albuali WH, Al-Mulhim AS, Jresat I. Cardioprotective effect of cannabidiol in rats exposed to doxorubicin toxicity. Environ Toxicol Pharmacol. 2013;36(2):347-357. 
12. Saeed NM, El-Naga RN, El-Bakly WM, Abdel-Rahman HM, Salah Eldin RA, El-Demerdash E. Epigallocatechin-3-gallate pretreatment attenuates doxorubicin-induced cardiotoxicity in rats: A mechanistic study. Biochem Pharmacol. 2015;95(3):145-155.

13. El-Agamy DS, Abo-Haded HM, Elkablawy MA. Cardioprotective effects of sitagliptin against doxorubicin-induced cardiotoxicity in rats. Exp Biol Med. 2016;241(14):1577-1587.

14. Costa PM, Ferreira PM, Bolzani VS, et al. Antiproliferative activity of pristimerin isolated from Maytenus ilicifolia (Celastraceae) in human HL-60 cells. Toxicol In Vitro. 2008;22(4):854-863.

15. Tong L, Nanjundaiah SM, Venkatesha SH, Astry B, Yu H, Moudgil KD. Pristimerin, a naturally occurring triterpenoid, protects against autoimmune arthritis by modulating the cellular and soluble immune mediators of inflammation and tissue damage. Clin Immunol. 2014;155(2):220-230.

16. Deeb D, Gao X, Liu YB, Pindolia K, Gautam SC. Pristimerin, a quinonemethide triterpenoid, induces apoptosis in pancreatic cancer cells through the inhibition of pro-survival Akt/NF- $\mathrm{\kappa B} / \mathrm{mTOR}$ signaling proteins and anti-apoptotic Bcl-2. Int J Oncol. 2014;44(5):1707-1715.

17. El-Agamy DS, Shaaban AA, Almaramhy HH, Elkablawy S, Elkablawy MA. Pristimerin as a novel hepatoprotective agent against experimental autoimmune hepatitis. Front Pharmacol. 2018;9:292.

18. Arafa MH, Mohammad NS, Atteia HH, Abd-Elaziz HR. Protective effect of resveratrol against doxorubicin-induced cardiac toxicity and fibrosis in male experimental rats. J Physiol Biochem. 2014;70(3):701-711.

19. Varghese F, Bukhari AB, Malhotra R, De A. IHC Profiler: an open source plugin for the quantitative evaluation and automated scoring of immunohistochemistry images of human tissue samples. PLoS One. 2014;9(5):e96801.

20. Abo-Haded HM, Elkablawy MA, Al-Johani Z, Al-Ahmadi O, El-Agamy DS. Hepatoprotective effect of sitagliptin against methotrexate induced liver toxicity. PLoS One. 2017;12(3):e0174295.

21. Pei XM, Yung BY, Yip SP, Ying M, Benzie IF, Siu PM. Desacyl ghrelin prevents doxorubicin-induced myocardial fibrosis and apoptosis via the GHSR-independent pathway. Am J Physiol Endocrinol Metab. 2014;306(3):E311-E323.

22. Wu X, Qi X, Lu Y, et al. Liguzinediol protects against cardiac fibrosis in rats in vivo and in vitro. Biomed Pharmacother. 2016;80:260-267.

23. Elsherbiny NM, Salama MF, Said E, El-Sherbiny M, Al-Gayyar MM. Crocin protects against doxorubicin-induced myocardial toxicity in rats through down-regulation of inflammatory and apoptic pathways. Chem Biol Interact. 2016;247:39-48.

24. Martinel Lamas DJ, Nicoud MB, Sterle HA, et al. Selective cytoprotective effect of histamine on doxorubicin-induced hepatic and cardiac toxicity in animal models. Cell Death Discov. 2015;1:15059.

25. Ezzat SM, El Gaafary M, El Sayed AM, et al. The cardenolide glycoside Acovenoside A affords protective activity in doxorubicin-induced cardiotoxicity in mice. $J$ Pharmacol Exp Ther. 2016;358(2):262-270.

26. Ma S, Li X, Dong L, Zhu J, Zhang H, Jia Y. Protective effect of ShengMai Yin, a traditional Chinese preparation, against doxorubicin-induced cardiac toxicity in rats. BMC Complement Altern Med. 2016;16:61.

27. Spinale FG, Coker ML, Bond BR, Zellner JL. Myocardial matrix degradation and metalloproteinase activation in the failing heart: a potential therapeutic target. Cardiovasc Res. 2000;46(2):225-238.

28. Polegato BF, Minicucci MF, Azevedo PS, et al. Acute doxorubicininduced cardiotoxicity is associated with matrix metalloproteinase-2 alterations in rats. Cell Physiol Biochem. 2015;35(5):1924-1933.

29. Zhang JM, An J. Cytokines, inflammation, and pain. Int Anesthesiol Clin. 2007;45(2):27-37.

30. Zhao W, Zhao T, Chen Y, Ahokas RA, Sun Y. Oxidative stress mediates cardiac fibrosis by enhancing transforming growth factor-beta 1 in hypertensive rats. Mol Cell Biochem. 2008;317(1-2):43-50.
31. Morrow DA, Cannon CP, Jesse RL, et al. National academy of clinical biochemistry laboratory medicine practice guidelines: clinical characteristics and utilization of biochemical markers in acute coronary syndromes. Circulation. 2007;115(13):e356-e375.

32. Shi Y, Moon M, Dawood S, McManus B, Liu PP. Mechanisms and management of doxorubicin cardiotoxicity. Herz. 2011;36(4): 296-305.

33. Stěrba M, Popelová O, Vávrová A, et al. Oxidative stress, redox signaling, and metal chelation in anthracycline cardiotoxicity and pharmacological cardioprotection. Antioxid Redox Signal. 2013;18(8): 899-929.

34. Raj S, Franco VI, Lipshultz SE. Anthracycline-induced cardiotoxicity: a review of pathophysiology, diagnosis, and treatment. Curr Treat Options Cardiovasc Med. 2014;16(6):1-14.

35. Zhao Y, Miriyala S, Miao L, et al. Redox proteomic identification of HNE-bound mitochondrial proteins in cardiac tissues reveals a systemic effect on energy metabolism after doxorubicin treatment. Free Radic Biol Med. 2014;72:55-65.

36. Singh P, Sharma R, McElhanon K, et al. Sulforaphane protects the heart from doxorubicin-induced toxicity. Free Radic Biol Med. 2015;86: $90-101$.

37. Yarmohmmadi F, Rahimi N, Faghir-Ghanesefat H, et al. Protective effects of agmatine on doxorubicin-induced chronic cardiotoxicity in rat. Eur J Pharmacol. 2017;796:39-44.

38. Hui B, Yao X, Zhou Q, Wu Z, Sheng P, Zhang L. Pristimerin, a natural anti-tumor triterpenoid, inhibits LPS-induced TNF- $\alpha$ and IL- 8 production through down-regulation of ROS-related classical NF- $\mathrm{KB}$ pathway in THP-1 cells. Int Immunopharmacol. 2014;21(2):501-508.

39. Johnson GL, Lapadat R. Mitogen-activated protein kinase pathways mediated by ERK, JNK, and p38 protein kinases. Science. 2002;298(5600):1911-1912.

40. Das J, Ghosh J, Manna P, Sil PC. Taurine suppresses doxorubicintriggered oxidative stress and cardiac apoptosis in rat via up-regulation of PI3-K/Akt and inhibition of p53, p38-JNK. Biochem Pharmacol. 2011;81(7):891-909.

41. Ghosh J, Das J, Manna P, Sil PC. The protective role of arjunolic acid against doxorubicin induced intracellular ROS dependent JNK-p38 and p53-mediated cardiac apoptosis. Biomaterials. 2011;32(21): 4857-4866.

42. Xiao J, Sun GB, Sun B, et al. Kaempferol protects against doxorubicininduced cardiotoxicity in vivo and in vitro. Toxicology. 2012;292(1): $53-62$.

43. Jin Y, Wang Y, Zhao D, Ma S, Lu J, Shuang G. Pristimerin attenuates ovalbumin-induced allergic airway inflammation in mice. Immunopharmacol Immunotoxicol. 2016;38(3):221-227.

44. Mukherjee S, Banerjee SK, Maulik M, Dinda AK, Talwar KK, Maulik SK. Protection against acute adriamycin-induced cardiotoxicity by garlic: role of endogenous antioxidants and inhibition of TNF-alpha expression. BMC Pharmacol. 2003;3:16.

45. Guerra J, De Jesus A, Santiago-Borrero P, Roman-Franco A, Rodríguez E, Crespo MJ. Plasma nitric oxide levels used as an indicator of doxorubicin-induced cardiotoxicity in rats. Hematol J. 2005;5(7):584-588.

46. Deng S, Kruger A, Schmidt A, et al. Differential roles of nitric oxide synthase isozymes in cardiotoxicity and mortality following chronic doxorubicin treatment in mice. Naunyn Schmiedebergs Arch Pharmacol. 2009;380(1):25-34.

47. Mukhopadhyay P, Rajesh M, Bátkai S, et al. Role of superoxide, nitric oxide, and peroxynitrite in doxorubicin-induced cell death in vivo and in vitro. Am J Physiol Heart Circ Physiol. 2009;296(5):H1466-H1483.

48. Morgan MJ, Liu ZG. Crosstalk of reactive oxygen species and NF- $\mathrm{kB}$ signaling. Cell Res. 2011;21(1):103-115. 


\section{Supplementary material}

Table SI PCR efficiency and melting temperature $(T m)$ of PCR primers used in this study

\begin{tabular}{|l|l|l|}
\hline Gene & PCR efficiency & $\mathbf{T}_{\mathbf{m}}\left({ }^{\circ} \mathbf{C}\right)$ \\
\hline TGF- $\beta$ I & 104.9 & 83 \\
\hline MMP-2 & 109.9 & 85.5 \\
\hline MMP-9 & 105.2 & 89.5 \\
\hline TIMP-I & 95.1 & 87 \\
\hline Fibronectin & 109.2 & 85.5 \\
\hline Colla I & 107.6 & 88.5 \\
\hline Nrf2 & 107.9 & 83.5 \\
\hline GCLc & 105.1 & 80 \\
\hline GCLm & 96.5 & 82.5 \\
\hline NQOI & 103.5 & 84.2 \\
\hline HO-I & 98.6 & 84 \\
\hline
\end{tabular}

Abbreviations: Coll $\alpha$ I, collagen type I alpha I; HO-I, heme-oxygenase-I; MMP2, metalloproteinase-2; MMP-9, metalloproteinase-9; GCLc, glutamatecysteine ligase catalytic subunit; GCLm, glutamate-cysteine ligase modifier; NQOI, NAD $(P)$ $\mathrm{H}$ dehydrogenase quinone; Nrf2, nuclear erythroid 2-related factor 2; TGF- $\beta$ I, transforming growth factor-betal; TIMP-I, tissue inhibitor of metalloproteinase-I.

Cancer Management and Research is an international, peer-reviewed open access journal focusing on cancer research and the optimal use of preventative and integrated treatment interventions to achieve improved outcomes, enhanced survival and quality of life for the cancer patient The manuscript management system is completely online and includes

\section{Dovepress}

a very quick and fair peer-review system, which is all easy to use. Visit http://www.dovepress.com/testimonials.php to read real quotes from published authors. 